Ophthalmologe 2022 $\cdot 119: 110-111$

https://doi.org/10.1007/s00347-021-01567-2

Angenommen: 21. Dezember 2021

(C) The Author(s), under exclusive licence to Springer Medizin Verlag GmbH, ein Teil von Springer Nature 2022

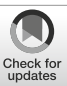

\section{Smartphone-basierte Fundusfotografie: eine zukunftsfähige Lösung?}

Maximilian W. M. Wintergerst

Klinik für Augenheilkunde, Universitätsklinikum Bonn, Bonn, Deutschland
Die Entwicklung von Geräten zur Fundusfotografie vor etwa 100 Jahren ermöglichte erstmals eine über die händische Zeichnung hinausgehende, objektive Befunddokumentation von Erkrankungen des hinteren Augenabschnittes. Dies beflügelte den wissenschaftlichen Diskurs und schaffte die Voraussetzungen für unsere moderne, bildgebungsbasierte Diagnostik und Verlaufsbeobachtung einer Vielzahl ophthalmologischer Erkrankungen. Seit ihrer Entwicklung ist die Durchführung der Fundusfotografie jedoch nur mit hoch spezialisierten Geräten möglich gewesen, was deren Anwendung - zumindest im ressourcenbeschränkten Kontext - oft einschränkte.

Die Smartphone-basierte Fundusfotografie hat uns nun die Möglichkeit der niedrigschwelligen Befunddokumentation v. a. im Hinblick auf den finanziellen Aufwand, die Delegierbarkeit und die Mobilität eröffnet und verfügt standardmäßig über Möglichkeiten zur Datenvernetzung und-verarbeitung. Da die Umsetzung vieler grundlegender ophthalmologischer Versorgungsprinzipien in Ländern mit niedrigem und mittlerem Einkommen sehr oft an begrenzten finanziellen und personellen Ressourcen scheitert, ist die Relevanz der Smartphone-basierten Fundusfotografie in diesem Kontext besonders hoch. Aber auch in Ländern mit hohem Einkommen existieren interessante Anwendungsmöglichkeiten.

\section{I) Smartphone-basierte Fundusfotografie eröffnet die Möglichkeit der niedrigschwelligen Befunddokumentation}

Folgende Fragen zum Thema Smartphonebasierte Fundusfotografie, die Sie sich si- cherlich bereits gestellt haben, werden wir im Rahmen dieses Leitthemas versuchen zu beantworten:

- Welche Geräte gibt es, und wie unterscheiden sie sich hinsichtlich Bildqualität, Bildfeld, Anschaffungskosten, verwendeter Technik, Gewicht, verfügbarer Software, SmartphoneKompatibilität und Zertifizierung?

- Worin unterscheidet sich die Smartphone-basierte Fundusfotografie technisch von konventionellen Geräten zur Fundusfotografie?

- Wie ist die medizinprodukterechtliche Situation, und welche Voraussetzungen zur Zertifizierung gibt es? Welche wichtigen Punkte gilt es für Sie, rechtlich und bezüglich der IT-Sicherheit zu beachten, wenn Sie Smartphonebasierte Fundusfotografie in Ihrer Praxis, Ihrer Klinik oder Ihrem Projekt anwenden wollen?

Auch wenn das Potenzial der Smartphonebasierten Fundusfotografie groß ist, gibt es Probleme, deren Lösbarkeit sich in Zukunft zeigen muss. Hierzu gehören z. B. die Kompatibilität der Adapter mit immer kürzeren Produktlebenszyklen der einzelnen Smartphone-Modelle und der Umgang mit Smartphones, die über mehrere Kameras verfügen.

Im Rahmen dieses Leitthemas werden Herr Jansen (Universitätsaugenklinik Bonn) et al. Ihnen im ersten Beitrag einen Überblick zu Adaptern und Anwendungen der Smartphone-basierten Fundusfotografie geben. Anschließend beleuchten Herr Dr. Jochen Straub und Herr Robert Sprowl (Carl Zeiss Meditec) die technischen Unterschiede zwischen Smartphone-basierter und konventionel- 
ler Fundusfotografie. Abschließend geben Herr Dr. Wolfgang Lauer (Bundesinstitut für Arzneimittel und Medizinprodukte [BfArM]), Frau Dr. Nicole Rämsch-Günther (ebenfalls BfArM) und Frau Dr. Dina Truxius (Bundesamt für Sicherheit in der Informationstechnik) in ihrem Beitrag eine medizinprodukterechtliche und ITsicherheitsbezogene Einschätzung zur Smartphone-basierten Fundusfotografie.

Die Smartphone-basierte Fundusfotografie hat in Ländern mit niedrigem und mittlerem Einkommen das Potenzial, die Verfügbarkeit ophthalmologischer Diagnostik zu revolutionieren. Vor diesem Hintergrund, aber auch angesichts eines möglichen Einsatzes in Ländern mit hohem Einkommen wie Deutschland wollen wir Ihnen einen Überblick zum aktuellen Stand und der möglichen zukünftigen Entwicklung geben.

Ich bedanke mich herzlich bei den Autoren und wünsche Ihnen eine anregende Lektüre!

Ihr

Maximilian W.M. Wintergerst

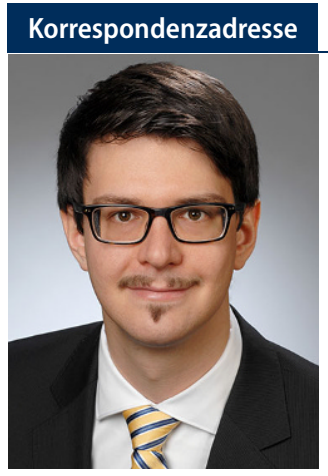

Dr. med. Maximilian W. M. Wintergerst

Klinik für Augenheilkunde, Universitätsklinikum Bonn

Ernst-Abbe-Str. 2, 53127 Bonn, Deutschland maximilian.wintergerst@ukbonn.de

Förderung. BONFOR GEROK Program, Faculty of Medicine, University of Bonn (Förder-Nr. 0-137.0028), Else Kröner-Fresenius Stiftung, Bundesministerium für Wirtschaftliche Zusammenarbeit und Entwicklung (BMZ), Novartis, CenterVue SpA, Berlin-Chemie AG, Deutsche Ophthalmologische Gesellschaft (DOG), Heine Optotechnik GmbH.

Interessenkonflikt. M.W.M. Wintergerst: Finanzielle Förderung: ONFOR GEROK Program, Faculty of Medicine, University of Bonn (Förder-Nr. 0 137.0028), Else Kröner-Fresenius Stiftung, Bundesministerium für Wirtschaftliche Zusammenarbeit und Entwicklung (BMZ), Novartis, CenterVue SpA, Berlin-Chemie AG, Deutsche Ophthalmologische Gesellschaft (DOG), Heine Optotechnik GmbH.

Beratertätigkeit: Heine Optotechnik GmbH; Honorare für Referententätigkeit: ASKIN \& CO GmbH, Heine Optotechnik GmbH; Reisekostenförderung: ASKIN \& CO GmbH, Berlin-Chemie AG, DigiSight Technologies, Heine Optotechnik GmbH, European Society of Retina Specialists (EURETINA), Deutscher Akademischer Austauschdienst (DAAD), Association for Research in Vision and Ophthalmology (ARVO); kostenlose Zurverfügungstellung von Material: Heidelberg Engineering, Optos, Carl Zeiss Meditec, CenterVue SpA, D Eye Srl, Heine Optotechnik $\mathrm{GmbH}$; kostenlose Bildanalysen: Eyenuk, Inc.

\section{Terminankündigung}

\section{2}

AAD 2022 hybrid

Düsseldorf, 16.03. - 19.03.2022

https://aad-kongress.de/

Jahrestagung der Bielschowsky-

Gesellschaft 2022

Freiburg i.B., 13.05. - 14.05.2022

http://www.bg2022.de/

\section{EUNOS 2022}

15th meeting of European Neuroophthalmology Society

Birmingham (UK), 20.06. - 23.06.2022

https://www.eunosweb.eu/

DOG 2022

Berlin, 29.09. - 02.10.2022

https://dog-kongress.de/

\section{3}

AAD 2023

Düsseldorf, 21.03. - 25.03.2023

https://aad-kongress.de/

DOG 2023

Berlin, 28.09. - 01.10.2023

https://dog-kongress.de/

2024

AAD 2024

Düsseldorf, 12.03. - 16.03.2024

https://aad-kongress.de/

DOG 2024

Berlin, 10.10. - 13.10.2024

https://dog-kongress.de/ 